\title{
Molecular docking study of sappan wood extract to inhibit PBP2A enzyme on methicillin-resistant Staphylococcus aureus (MRSA)
}

\author{
${ }^{1}$ Department of Biology Pharmacy, Faculty of Pharmacy, University of Surabaya, Jl. Raya Kalirungkut, Surabaya, East Java \\ 60293, Indonesia, Phone: +62 31 2981110, E-mail: mariscaevalina@gmail.com \\ ${ }^{2}$ Department of Biology Education, Indonesia University of Education, Bandung, West Java, Indonesia
}

\begin{abstract}
:
Background: PBP2a is a type of penicillin-binding proteins (PBPs) that cause resistivity in methicillin-resistant Staphylococcus aureus (MRSA) from $\beta$-lactam antibiotics. MRSA susceptible with cefttobiprole (fifth generation of cephalosporin as an anti-MRSA agent) which inhibits PBP2a and stops its growth. Contrary to its efficacy, ceftobiprole causes taste disturbance more than any other cephalosporins; furthermore, its mechanism is unknown. This study aims to explore an in silico study of a natural compound, which serves as a potential alternative to overcome MRSA with minimum adverse side effects.

Methods: A molecular docking study was performed using Molegro Virtual Docker version 5.5. Brazilin and proto-sappanins A-E are phytochemical compounds contained in sappan wood extract and are docked into the binding site of PBP2a (Protein Data Bank: ID 4DKI).

Results: Brazilin and proto-sappanins A-E have some interaction with Ser 403 amino acid residue which is an important interaction to inhibit PBP2a protein. The result of the molecular docking study showed that the MolDock score of proto-sappanins D and $\mathrm{E}$ is lower than that of methicillin but higher than that of its native ligand (ceftobiprole).

Conclusions: The results of this study suggest that proto-sappanins $\mathrm{D}$ and $\mathrm{E}$ have an excellent potential activity as an alternative to ceftobiprole in limiting MRSA growth through PBP2A enzyme inhibition.
\end{abstract}

Keywords: antimicrobial, molecular docking, MRSA, PBP2a, sappan wood

DOI: $10.1515 /$ jbcpp-2019-0282

Received: September 25, 2019; Accepted: December 13, 2019

\section{Introduction}

Methicillin-resistant Staphylococcus aureus (MRSA) is a strain of gram-positive bacteria that is genetically different from the other S. aureus [1]. MRSA causes life-threatening bloodstream infections, pneumonia, and surgical site infections in humans at health care facilities. Hospital-acquired infections caused by MRSA are often systemic infections, whereas community-acquired MRSA infections are skin and soft tissue infections [2]. These MRSA bacteria are developed by horizontal transfer gene or multiple drug resistance to $\beta$-lactam antibiotics, including penicillin derivate (methicillin or oxacillin), cephalosporin, or carbapenem [3]. MRSA resistance is caused by structural modification of penicillin-binding proteins (PBPs) [4], [5]. The mecA gene is a biomarker responsible gene found in bacteria, which causes resistance to methicillin and other $\beta$-lactam antibiotics. The mecA gene encodes is PBP2a, which differs from other PBPs that do not allow methicillin and other $\beta$-lactam antibiotics bind to their active site [1], [6], [7].

Ceftobiprole is a broad-spectrum antibiotic which shows activity against MRSA [8]. Ceftobiprole is the frontrunner of the fifth cephalosporin generation, which is used as a comparator drug to an alternative natural compound with potential anti-MRSA activity. Although some $\beta$-lactam antibiotics in the fifth-generation broad spectrum show their activity against MRSA, their adverse side effects cause limitation to their usage as comparator drugs [3], [9]. Natural compound with anti-microbial effects in search for alternative drugs against MRSA. This study aims to explore natural compounds which serve as a potential alternative to overcome MRSA with minimum adverse side effects.

Sappan wood extract is known to have anti-microbial properties. It has been tested against different microorganisms for its potential anti-microbial activity, including S. aureus [10]. The investigation of the chemical 
constituents of sappan wood extract shows that it has various types of phenolic compounds including brazilin and proto-sappanin [11], [12]. Hence, in this study a natural compound from sappan wood extract was analyzed for its effectiveness with respect to the existing drugs (ceftobiprole) against MRSA through in silico studies (molecular docking) using Molegro Virtual Docker (MVD) version 5.5.

\section{Materials and methods}

\section{Receptor and ligand preparation}

PBP2a receptors obtained from Protein Data Bank (PDB) were prepared using the MVD version 5.5 software, as shown in Figure 1. PBP is an enzyme that catalyzes processes of the building cell wall process of some bacteria. PBP2a is a type of PBP that causes resistivity in MRSA from $\beta$-lactam antibiotics. PBP2a was downloaded with PDB ID code 4DKI from www.rcsb.org [13]. The comparative ligand from PBP2a receptor is ceftobiprole (its native ligand) from the x-ray crystallography was downloaded and stored in the form of mol.files for re-docking in order to validate the MVD program, used to docked the brazilin and proto-sappanins A-E (they are phytochemical compounds contained in sappan wood extract). Before the docking process, brazilin and proto-sappanin A-E structures were built with ChemBioDraw Ultra 11.0 and their geometry optimizations were performed using MMFF94 [14]. The phytochemical compounds from sappan wood extract were docked into the active site of PBP2a receptors that have the active site occupied by their native ligand ceftobiprole, as shown in Figure 1.

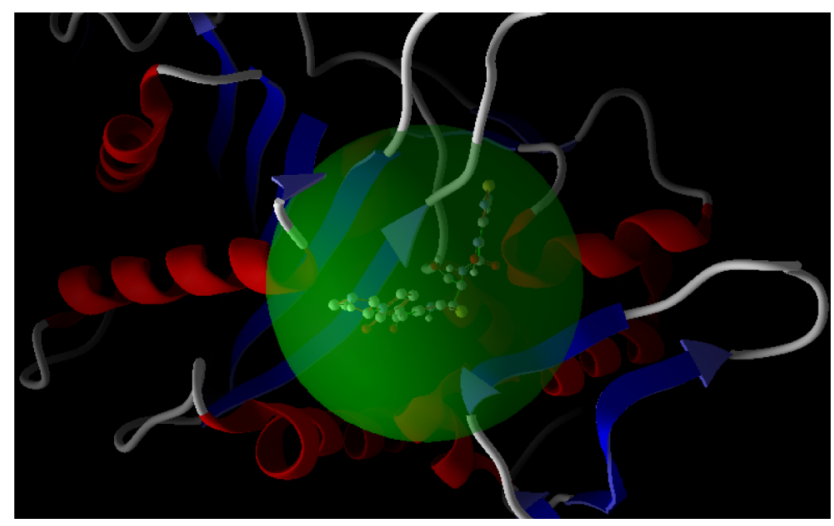

Figure 1: Active site of the PBP2a receptor.

\section{Ligand receptor docking study and visualization of docking results}

The docking is carried out with the native ligand, brazilin, and proto-sappanins A-E using MVD version 5.5. The result of Molecular docking study was evaluated using the MolDock score as scores interpreted as predictions of bond interactions between ligand and receptors.

The MolDock score is the total energy from external ligand interaction plus internal ligand interaction. External ligand interaction is the sum of energy consisting of protein-ligand interaction and cofactor-ligand interaction. Internal energy interaction is the sum of energy dependent on the chemical structure of the ligand such as torsional strain, torsional strain $\mathrm{sp}^{2}-\mathrm{sp}^{2}$, steric, and electrostatic. The MolDock score formula is as shown in Figure 2. 


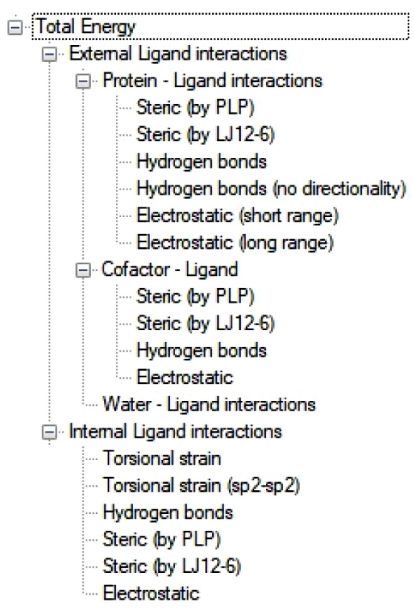

$$
\begin{aligned}
& E_{\text {score }}=E_{\text {inter }}+E_{\text {intra }} \\
& E_{\text {inter }}=\sum_{i \in \text { ligand }} \sum_{j \in \text { protein }}\left[E_{P L P}\left(r_{i j}\right)+332.0 \begin{array}{c}
q_{i} q_{J} \\
4 r_{i j}^{2}
\end{array}\right] \\
& E_{\text {inter }} \text { is the internal energy of the ligand: } \\
& E_{\text {inra }}=\sum_{\text {ieligand }} \sum_{\text {eligand }} E_{P L P}\left(r_{i j}\right)+\sum_{\text {fexible bonds }} A\left[1-\cos \left(m \cdot \theta-\theta_{0}\right)\right]+E_{\text {clash }}
\end{aligned}
$$

Figure 2: Formula of the MolDock score.

The lower MolDock score indicates that smaller amount of energy is required for forming the ligandreceptor interaction. The MolDock score interprets all accumulation energy between the ligand-receptor interaction, which includes hydrogen bond, steric interaction, and electronic interaction.

\section{Validation of the docking process}

Molecular docking of the drug to be tested was done in order to analyze the stability of the receptor protein. The in silico validation was carried out by re-docking the native ligand (ceftobiprole) into its active site. The criterion of acceptance is set with the value of root mean square deviation (RMSD) calculated for the protein's backbone atoms of less than $2.0 \AA$, as shown in Figure 3 .

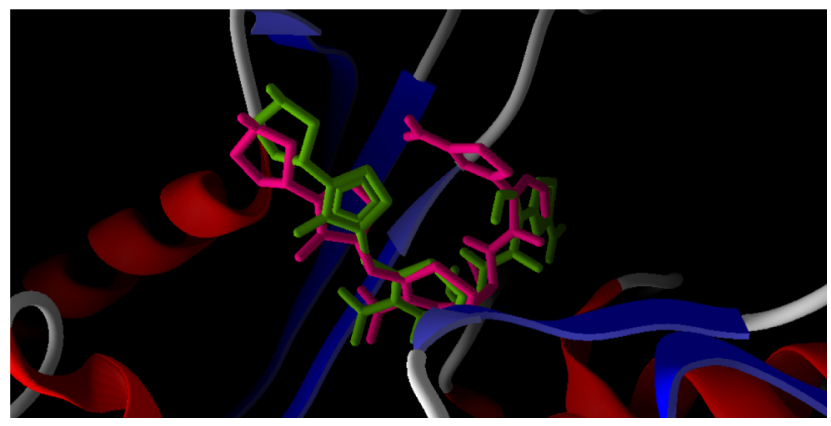

Figure 3: Comparison of native ligand (green) with docking result simulation (pink) by the Molegro Virtual Docker (MVD) software, version 5.5. The RMSD is $1.98 \AA$.

After the re-docking process, the brazilin and proto-sappanins A-E were docked into the active site of the PBP2a receptor. The binding affinity between the ligand and receptor (docking score) was evaluated using the MolDock score, and we also compared the scores of ceftobiprole as its native ligand and methicillin (as a standard microbiological activity against MRSA) with brazilin and proto-sappanins A-E.

\section{Results}

In this research for the molecular docking study, we docked brazilin and proto-sappanins A-E (phytochemical compounds from sappan wood extract) compared with methicillin and ceftobiprole (native ligand from PBP2a). The docking result is shown in Table 1 and Figure 4. The MolDock score of proto-sappanin D ( -144.21 $\mathrm{kcal} / \mathrm{mol})$ and $\mathrm{E}(-146.48 \mathrm{kcal} / \mathrm{mol})$ is lower than that of methicillin $(-119.50 \mathrm{kcal} / \mathrm{mol})$, but higher than that of ceftobiprole (-171.14 kcal/mol), an anti-MRSA agent; moreover, the MolDock score of proto-sappanins A-C and brazilin is higher than that of methicillin and ceftobiprole. The comparison of the native ligand is shown in Figure 3. 


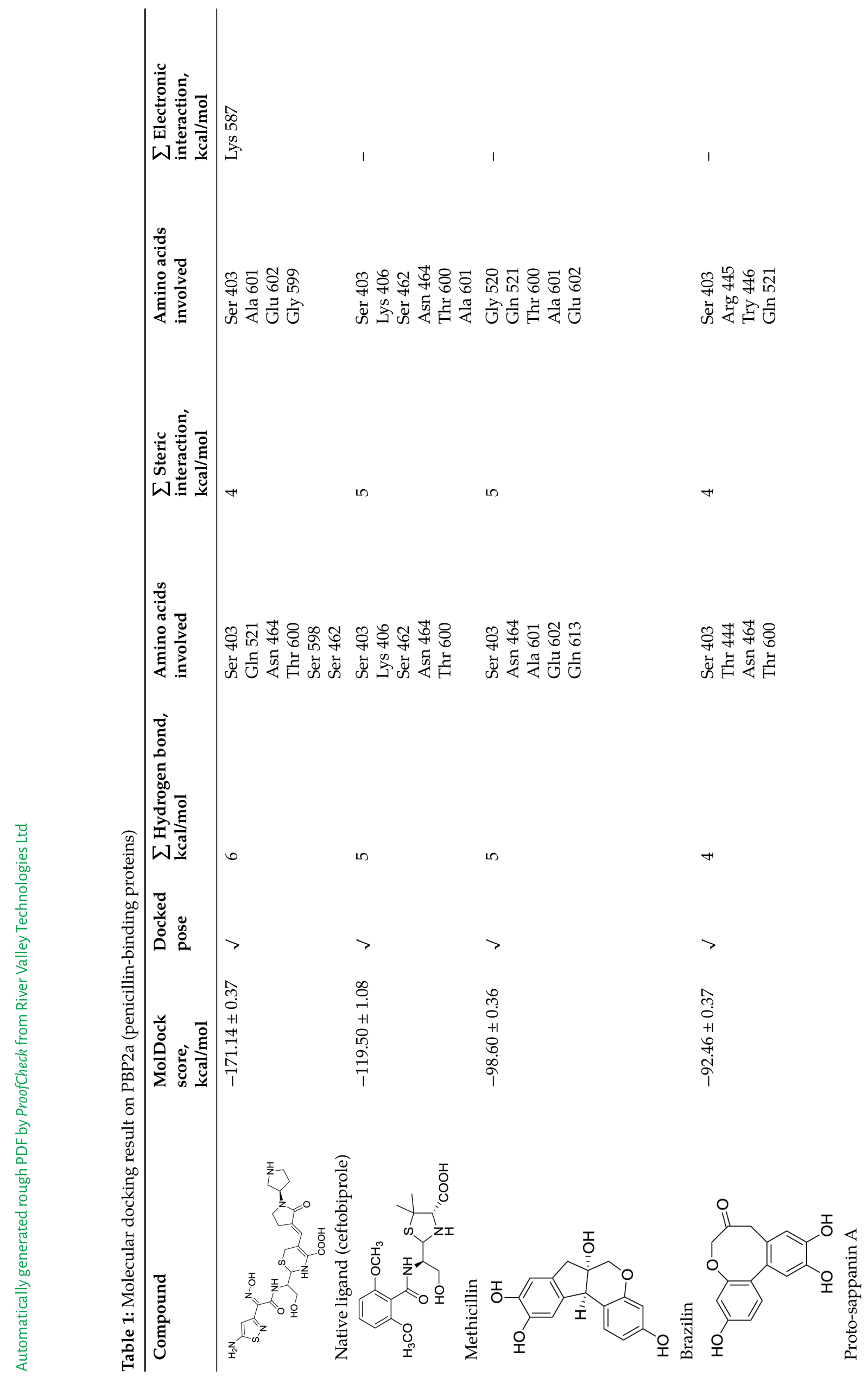




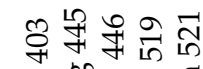

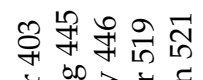

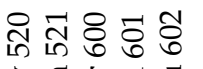

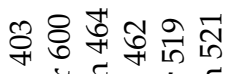

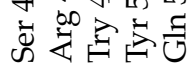

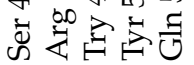

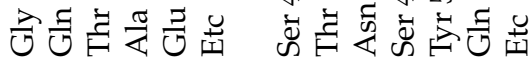

เ

เ

$\stackrel{N}{\sim}$

$a$

위연

㚔苯贲 8

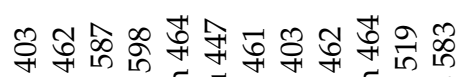

离㤩要

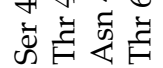

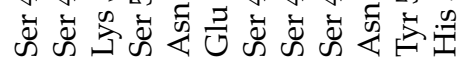

$\infty$

เ

$\begin{array}{ll}1 & 5 \\ +1 & 0 \\ +1 & +1 \\ +1 & 10 \\ 0 & 0 \\ 0 & 0 \\ 0 & 0 \\ 1 & 0\end{array}$

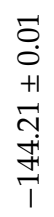

-
0
0
+1
$\infty$
+1
0
+1
1

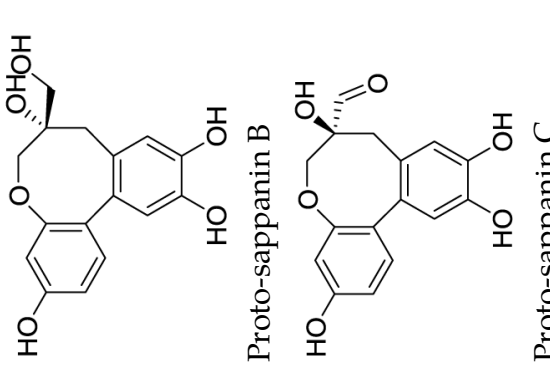

I

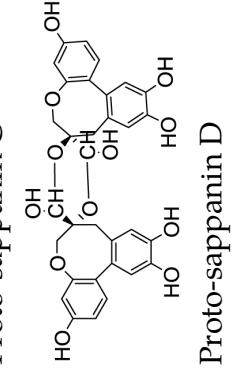

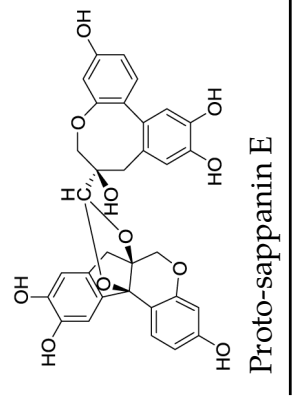




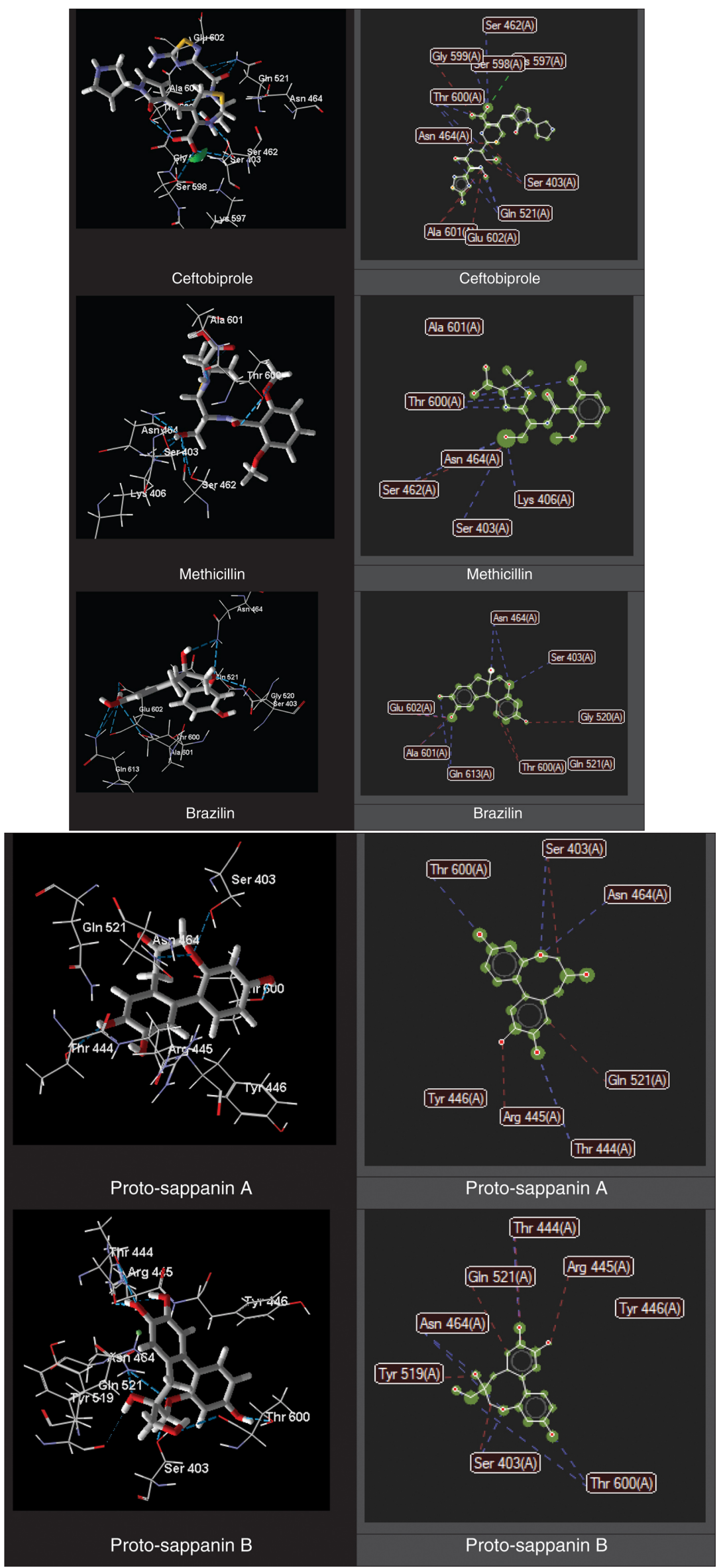




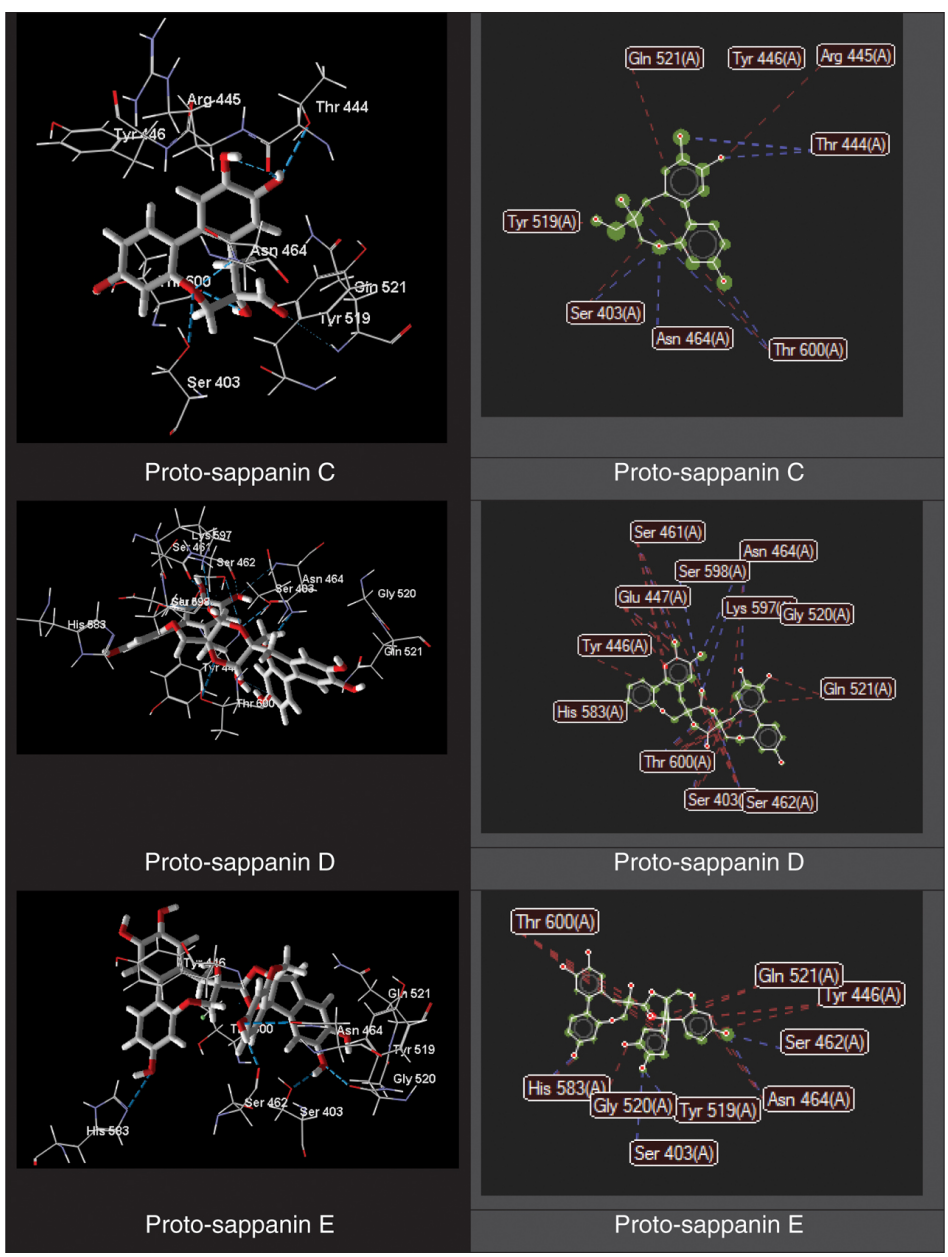

Figure 4: The interaction between its native ligand ceftobiprole, methicillin, brazilin, and proto-sappanins A-E into the active site of PBP2a.

\section{Discussion}

Brazilin and proto-sappanins A-E were docked into the active site of PBP2a. This enzyme was chosen because it catalyzes the cell wall of bacteria that had been resistant to methicillin, which we called MRSA. PBP2a is an enzyme transpeptidase that catalyzes bacterial wall processes as shown in Figure 5, especially in gram-positive bacteria, which generally contain peptidoglycan.
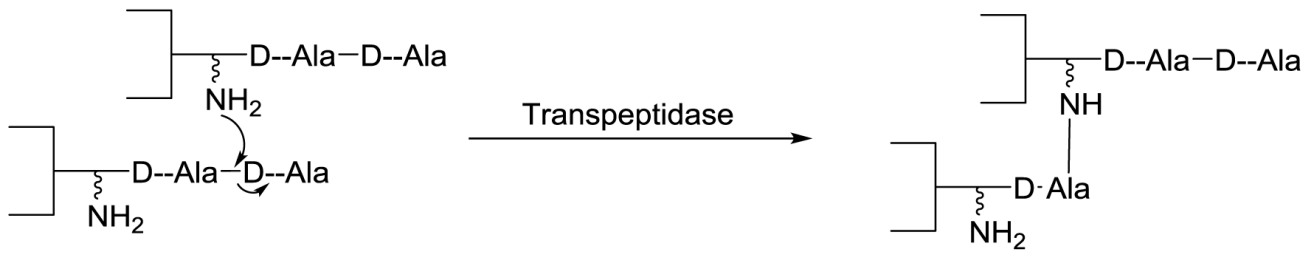

peptidoglycan (cells wall of bacteria)

Figure 5: Transpeptidase that catalyzes bacterial wall processes by cross-linking.

PBP2a is highly resistant to methicillin. Methicillin is a standard antibiotic which indicates that bacteria are still sensitive or resistant to the $\beta$-lactam antibiotic groups.

Methicillin is not used in medical treatment because of its nephrotoxic side effects, while ceftobiprole is a fifth-generation cephalosporin antibiotic that can be an anti-MRSA agent.

Based on molecular docking result shown in Table 1, we find that ceftobiprole has the lowest MolDock score because of its accumulation energy from hydrogen bond (in amino acid residues such as Ser 403, Gln 521, Asn 464, Thr 600, Ser 598, Ser 462), steric interaction (on amino acid residues Ser 403, Ala 601, Glu 602, Gly 599), 
and electronic interaction on Lys 587 residue, which shows that the least energy is needed by ceftobiprole to interact with PBP2a.

Based on the molecular docking data, the most important amino acid residue on the process of opened $\beta$ lactam ring is Ser 403. PBP2a inactivation directly depends on covalent bond with serin residue on the process of opened $\beta$-lactam ring. Oxocan-3-one ring of proto-sappanins A-C and pyran ring of brazilin have some interactions with Ser 403 residue. The interaction of Proto-sappanins A-C on active site PBP2a enzyme are weaker than methicillin and ceftobiprole because $\beta$-lactam ring is strongly reactive and in the molecular level, the mechanism of $\beta$-lactam ring is nucleophile attack from hydroxyl functional group from serin (transpeptidase) to carbonyl $\beta$-lactam ring followed by SN-acyl reaction and therefore inactivated biosynthesis of peptidoglycan, as shown in Figure 6.

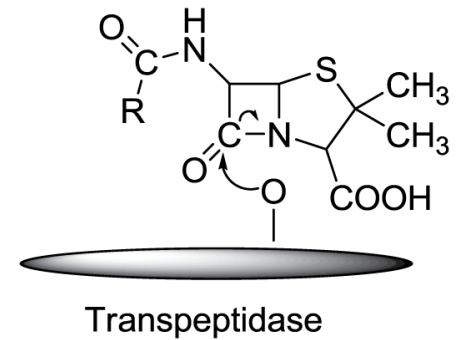

Transpeptidase

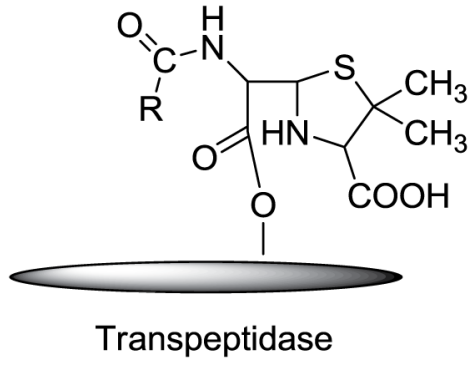

Figure 6: Inactivated transpeptidase by $\beta$-lactam ring.

The interaction of proto-sappanins A-C different with proto-sappanins D and E, both have condensated ring between another proto-sappanin or with brazilin. The condensated ring is easier to be the opened ring and interaction with Ser 403 residue than oxocan-3-one and pyran ring; therefore, the MolDock score of protosappanins $\mathrm{D}$ and $\mathrm{E}$ is lower than that of methicillin but higher than that of ceftobiprole. Finally, to prove the proto-sappanin D and E activity against MRSA, it is necessary to perform isolation and in vitro antibacterial test against MRSA strains.

\section{Conclusions}

Proto-sappanins D and E are predicted to have an activity that inhibits the growth of MRSA better than methicillin but not as well as ceftobiprole.

\section{Acknowledgment}

Special thanks to Prof. Dr. Siswandono, MS, Apt, from Airlangga University for MVD program licensed.

Research funding: None declared.

Author contributions: All authors have accepted responsibility for the entire content of this manuscript and approved its submission.

Competing interests: The authors state no conflict of interest.

\section{References}

[1] Llarrull LI, Fisher JF, Mobashery S. Molecular basis and phenotype of methicillin resistance in Staphylococcus aureus and insights into new $\beta$-lactams that meet the challenge. Antimicrob Agents Chemother 2009;53:4051-63.

[2] Waness A. Revisiting methicillin-resistant Staphylococcus aureus infections. ] Clob Infect Dis 2010;2:49.

[3] Gajdács M. The continuing threat of methicillin-resistant Staphylococcus aureus. Antibiotics 2010;8:52.

[4] Fishovitz ], Hermoso ]A, Chang M, Mobashery S. Penicillin-binding protein 2a of methicillin-resistant Staphylococcus aureus. IUBMB Life 2014;66:572-7.

[5] Foxley MA, Friedline AW, Jensen JM, Nimmo SL, Scull EM, King JB, et al. Efficacy of ampicillin against methicillin-resistant Staphylococcus aureus restored through synergy with branched poly(ethylenimine). J Antibiot (Tokyo) 2016;69:871. 
[6] Graves-Woodward K, Pratt RF. Reaction of soluble penicillin-binding protein 2a of methicillin-resistant Staphylococcus aureus with $\beta$ lactams and acyclic substrates: kinetics in homogeneous solution. Biochem ] 1998:332:755-61.

[7] Gurusamy KS, Koti R, Toon CD, Wilson P, Davidson BR. Antibiotic therapy for the treatment of methicillin-resistant Staphylococcus aureus (MRSA) infections in surgical wounds. Cochrane Database Syst Rev 2013;8:CDo09726.

[8] Zhanel CG, Lam A, Schweizer F, Thomson K, Walkty A, Rubinstein E, et al. Ceftobiprole: a review of a broad-spectrum and anti-MRSA cephalosporin. Am ] Clin Dermatol 2008;9:245-54.

[9] Lovering AL, Gretes MC, Safadi SS, Danel F, Castro L, Page MG, et al. Structural insights into the anti-methicillin-resistant Staphylococcus aureus (MRSA) activity of ceftobiprole. ] Biol Chem 2012;287:32096-102.

[10] Srinivasan R, Karthik S, Mathivanan K, Baskaran R, Karthikeyan M, Gopi M, et al. In vitro antimicrobial activity of Caesalpinia sappan L. Asian Pac] Trop Biomed 2012;2:S136-9.

[11] Nirmal NP, Rajput MS, Prasad RG, Ahmad M. Brazilin from Caesalpinia sappan heartwood and its pharmacological activities: a review. Asian Pac ] Trop Med 2015;8:421-30.

[12] Zanin ]L, De Carvalho BA, Salles Martineli P, Dos Santos MH, Lago JH, Sartorelli Jr P, et al. The genus Caesalpinia L. (Caesalpiniaceae): phytochemical and pharmacological characteristics. Molecules 2012;17:7887-902.

[13] Thomas HA. Merck molecular force field. Basis, form, scope, parametrization, and performance of MMFF94. J Com Chem 1996;17:490519.

[14] Thomsen R, Christensen MH. MolDock: a new technique for high-accuracy molecular docking. ] Med Chem 2006;49:3315-21. 